\title{
A Novel Treatment for Massive Hemorrhage after Maternal Trauma in Pregnancy
}

\author{
Maritza G. Gonzalez, MD ${ }^{1}$ Ruth M. Wei, MD ${ }^{1}$ Kenneth D. Hatch, MD ${ }^{1}$ Lynn M. Gries, MD, FACS ${ }^{2}$ \\ Meghan G. Hill, MBBS ${ }^{1}$ \\ ${ }^{1}$ Department of Obstetrics and Gynecology, University of Arizona, \\ Tucson, Arizona \\ ${ }^{2}$ Department of Surgery, University of Arizona, Tucson, Arizona \\ Address for correspondence Maritza G. Gonzalez, MD, Department \\ of Obstetrics and Gynecology, University of Arizona, 1501 North \\ Campbell Avenue, 8th Floor 8327G, Tucson, AZ 85724 \\ (e-mail: mggonzalez@obgyn.arizona.edu).
}

Am J Perinatol Rep 2019;9:e27-e29.

\begin{abstract}
Keywords

- trauma

- pregnancy

- uterine compression

- disseminated intravascular coagulation

Background Trauma in pregnancy can lead to life-threatening hemorrhage. Conventional treatments of hemorrhage include medical and surgical management. However, if these measures fail uterine compression is an option to control bleeding. We present a case where this management was employed.

Case A patient presented at 36 weeks of gestation with multiple injuries after a motor vehicle collision and experienced disseminated intravascular coagulation (DIC). The use of a Bakri balloon in combination with external compression with Coban, a sterile selfadherent bandage, after delivery temporized her bleeding and allowed her to become stable for further management.

Conclusion When other measures fail and a hysterectomy is considered unsafe, the combination of internal and external uterine compression is an option.
\end{abstract}

\section{Introduction}

Trauma in pregnancy can lead to multiple complications and present life-threatening hemorrhage and disseminated intravascular coagulation, which can prove fatal. ${ }^{1-4}$ When caring for an unstable patient in the immediate postpartum period, often a hysterectomy is deemed necessary to resolve hemorrhage concerns. We present a case where a hysterectomy immediately postpartum would have proven fatal for the patient, however a combination of uterine tamponade with balloon catheter and external uterine compression allowed the patient time to help recover from the disseminated intravascular coagulopathy she was experiencing before definitive surgery was able to be performed safely.

\section{Case}

The patient is a 20-year-old G2P1001 at 36 weeks of gestation who presented after a motor vehicle collision. The patient was the unrestrained driver and was displaced within the car at the time of retrieval. Emergency department CT (computed tomography) scan revealed a splenic rupture, femoral fracture, pelvic fracture, rib fractures, lumbar spine fractures, multiple soft tissue injuries, facial injuries, and a fetal skull fracture (-Fig. 1). Fetal monitoring showed frequent contractions and a category III fetal tracing.

The patient was transferred to the operating room where a midline laparotomy and a low transverse cesarean delivery were performed and produced a compromised neonate (weight 3,280 grams, Apgar's scores: 1 at 1 minute, 6 at 5 minutes, and 8 at 10 minutes). After closure of the hysterotomy, the uterus contracted well but continued bleeding in spite of manual external compression which was applied continuously while the spleen was removed. A coagulopathy from massive hemorrhage was suspected when the peritoneal surfaces were noted to be bleeding during the unsuccessful attempts to achieve hemostasis. These techniques utilized included oversewing of the hysterotomy, placement of a Bakri balloon, and a unilateral uterine artery ligation suture. A hypogastric ligation was considered received

February 20, 2018

accepted after revision

September 24, 2018
DOI https://doi.org/

10.1055/s-0039-1678735. ISSN 2157-6998.
Copyright (c) 2019 by Thieme Medical Publishers, Inc., 333 Seventh Avenue, New York, NY 10001, USA. Tel: +1(212) 584-4662.
License terms

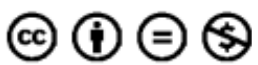




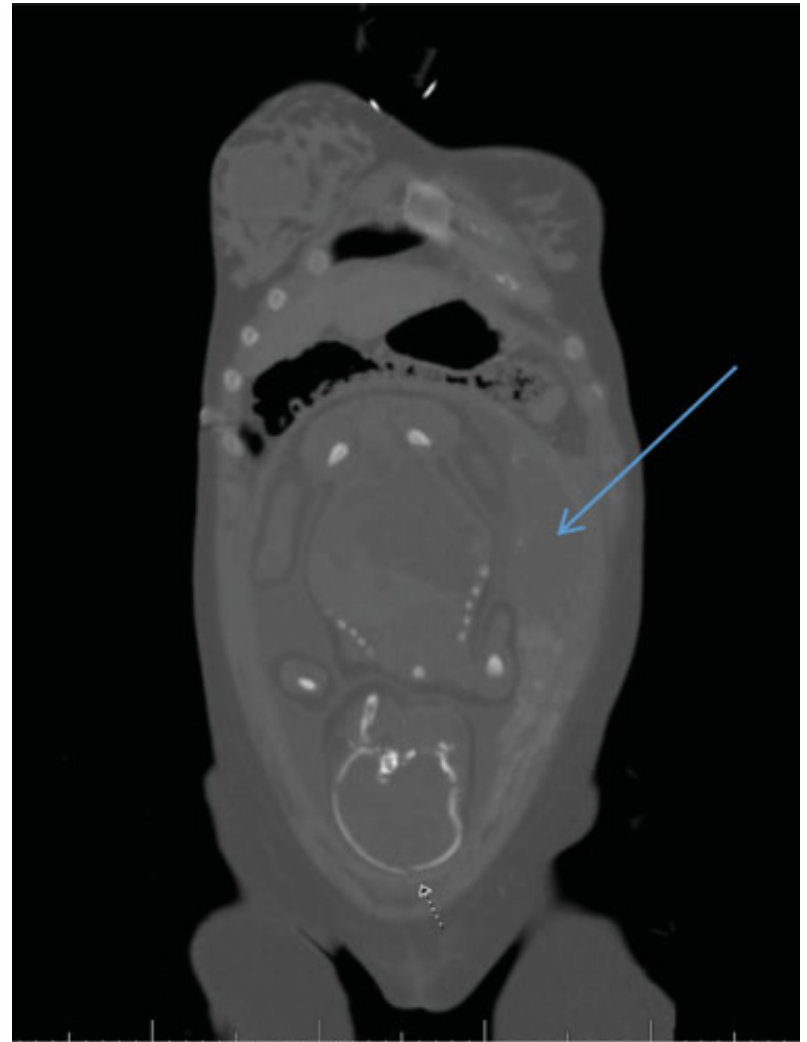

Fig. 1 CT of the pregnant uterus, note the fetal skull fracture (small arrow) and likely placental abruption (large arrow). CT, computed tomography.

but significant bleeding from the peritoneal surfaces was noted during dissection of the retroperitoneum, and the procedure was abandoned. The patient had already received approximately 36 units of balanced (1:1:1) blood products at this time as per our massive transfusion protocol. The surgeon felt the patient would not survive a hysterectomy. In addition to placement of a Bakri balloon, two separate sutures of VICRYL (polyglactin 910) woven mesh were externally placed around the uterus. The woven mesh was sewn tightly around the uterus to itself; it covered the lower uterine segment up to the level of the round ligament. Sufficient uterine compression was not obtained. Coban sterile self-adherent bandage was then used to firmly wrap the uterus in its entirety. Cessation of bleeding was noted at this time (-Fig. 2). Application was straightforward and circumferential compression occurred with ease. Sterile self-adherent bandages are readily available in operating departments that routinely perform vascular and orthopedic procedures.

The patient had an Rh-negative blood type and an antibody to $\mathrm{D}$ of unknown origin. Massive transfusion protocol continued postoperatively with profuse bleeding being noted from her abdomen, IV sites, nose, and mouth. The hospital's Rh-negative blood was depleted and the patient was continuing to bleed profusely, so the decision was made to give Rh-positive blood while negative blood was couriered. The patient did not experience a transfusion reaction,

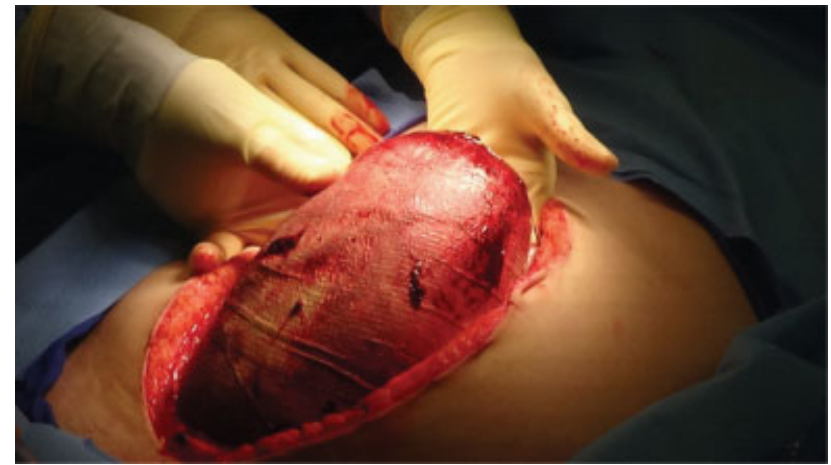

Fig. 2 Uterus with VICRYL (polyglactin 910) woven mesh (not seen as it is the internal layer) and Coban sterile adherent bandage used to wrap.

and records later confirmed that the antibody was likely from her receipt of Rhogam earlier in the pregnancy. Patient received approximately 100 units of balanced blood products during her initial hospitalization.

Her postoperative course was complicated. Over the next several days, the patient underwent interventional radiology vascular embolization to multiple sites, with significant bleeding noted into the buttocks from her pelvic fracture. There was never bleeding noted from the uterus during these procedures; however, the uterine arteries were embolized empirically on postoperative day 0 after initial laparotomy and again on postoperative day 3. Additionally, the patient was taken back to the operating room for continued abdominal bleeding less than 12 hours from her initial surgery. Upon entry into the abdomen, the bleeding (initially assumed to be from the uterus) was found to be coming from bleeding surfaces in multiple locations including a large subscapular hepatic hematoma, peritoneal surfaces, and vascular pedicles confirming coagulopathy. The uterus was

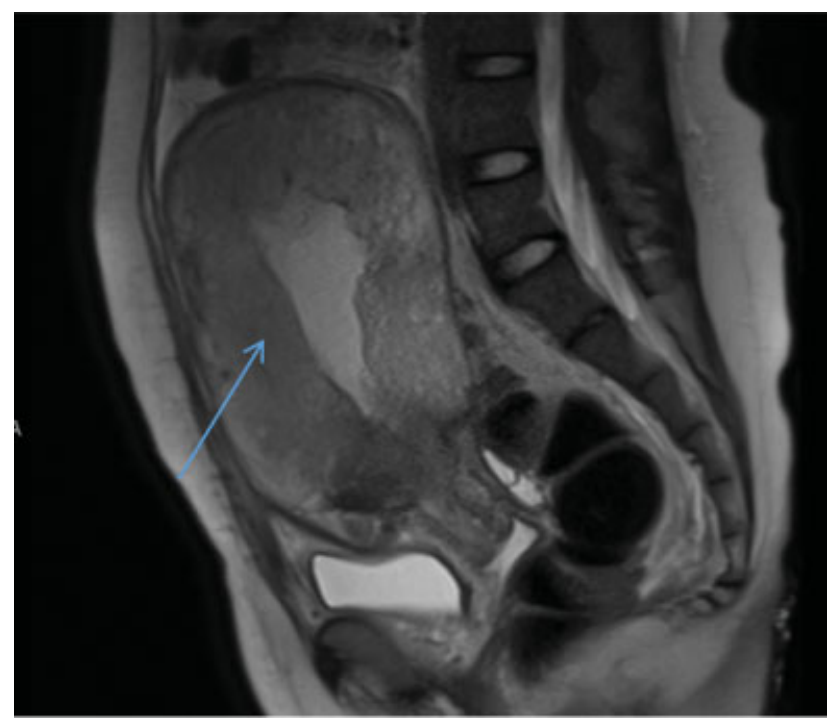

Fig. 3 MRI abdomen and pelvis on postoperative day 24, revealing myometrial necrosis demonstrated by lack of filling with contrast (arrow). MRI, magnetic resonance imaging. 
noted to be hemostatic at the end of this procedure. The patient had multiple areas of peritoneum oversewn and treated with Surgicel and Gelfoam and the liver was packed. Two days after the patient's initial procedures, the patient was reoperated upon to remove the lap sponges around the liver. The Coban and VICRYL (polyglactin 910) woven mesh were removed from the uterus at this time. The uterus remained hemostatic; a hysterectomy was not deemed necessary. On postoperative day 2 from her initial procedures, the patient was reoperated upon to remove the lap sponges around the liver. In spite of prophylactic anticoagulation, the patient experienced a pulmonary embolism as well. An MRI (magnetic resonance imaging) done 24 days after the initial procedure confirmed infarcted myometrium and a hysterectomy was performed (-Fig. $\mathbf{3}$ ).

This report does not detail all of the operations and procedures that the patient underwent but does reflect the main details of her obstetric care. The patient ultimately made a good recovery and has been undergoing rehabilitation for multiple musculoskeletal injuries at the conclusion of our care.

\section{Discussion}

Trauma in pregnancy can lead to severe hemorrhage and disseminated intravascular coagulation (DIC) which can prove fatal. ${ }^{1-4}$ We present a case of life-threatening polytrauma with multiple sources of bleeding in a patient who arrived in DIC. The diagnosis of DIC was made clinically on entry into the uterus and was later confirmed by laboratory criteria. When standard conservative measures failed to control the patient's bleeding, a hysterectomy was considered but it was thought such an effort would prove fatal. A decision was made to attempt surgical bimanual compression of the uterus. We have not identified any other examples of a similar technique in the literature, nor have we identified an optimal approach for management of such cases. Most authors agree that aggressive fluid resuscitation and correction of coagulopathy be combined with surgical techniques where necessary. ${ }^{3-6}$

Though arguments can be made for pursuing a hysterectomy earlier in the course of management than was done in this patient, we feel there is still value in reporting this approach. When confronted with an unstable patient the best option may be medical stabilization which was thought to provide the best chance of survival in this case. ${ }^{6}$ In an attempt to temporize the patient's condition so she could be further stabilized, we decided to perform tight internal and external compression of the uterus with a Bakri balloon internally and Coban sterile self-adherent dressing externally. At the time, we were not concerned with preserving this young patient's uterus for future fertility but rather for her survival. As she recovered over the next several days, the question arose as to whether the uterine tissue could survive an extended amount of time with a Bakri balloon in place, external compression with Coban sterile self-adherent bandage, and the multiple embolizations. In this case, it did not, and the uterus was removed after the diagnosis of myometrial infarction was made. Although it is undesirable to subject a patient to an interval surgery for a hysterectomy, we suggest that it may be a temporizing option in cases of refractory hemorrhage in a moribund patient.

\section{Teaching points}

1. It is important to consider options other than continued surgical attempts to stop bleeding in patients with lifethreatening hemorrhage because patients in disseminated intravascular coagulation may not tolerate a hysterectomy.

2. A Bakri balloon in combination with external compression using sterile Coban could be considered.

Conflict of Interest

None.

\section{References}

1 Creanga AA, Berg CJ, Ko JY, et al. Maternal mortality and morbidity in the United States: where are we now? J Womens Health (Larchmt) 2014;23(01):3-9

2 Callaghan WM, Creanga AA, Kuklina EV. Severe maternal morbidity among delivery and postpartum hospitalizations in the United States. Obstet Gynecol 2012;120(05):1029-1036

3 Cunningham FG, Nelson DB. Disseminated intravascular coagulation syndromes in obstetrics. Obstet Gynecol 2015;126(05): 999-1011

4 Pearce C, Martin SR. Trauma and considerations unique to pregnancy. Obstet Gynecol Clin North Am 2016;43(04):791-808

5 Mannucci PM, Levi M. Prevention and treatment of major blood loss. N Engl J Med 2007;356(22):2301-2311

6 Hunt BJ. Bleeding and coagulopathies in critical care. N Engl J Med 2014;370(09):847-859 